\title{
Fusion of real-time 3D transesophageal echocardiography and cardiac fluoroscopy imaging in transapical catheter-based mitral paravalvular leak closure
}

\author{
Aleksejus Zorinas ${ }^{1}$, Vilius Janusauskas ${ }^{1}$, Giedrius Davidavicius ${ }^{1}$, Lina Puodziukaite ${ }^{1}$, Diana Zakarkaite ${ }^{1}$, \\ Rita Kramena ${ }^{1}$, Rasa Čypienè ${ }^{1}$, Valdas Bilkis ${ }^{1}$, Kestutis Rucinskas ${ }^{1}$, Audrius Aidietis ${ }^{1}$, Eustaquio M. Onorato \\ ${ }^{1}$ Department of Cardiovascular Medicine, Vilnius University, Vilnius, Lithuania \\ ${ }^{2}$ Cardiovascular Department, Humanitas Gavazzeni, Bergamo, Italy
}

Adv Interv Cardiol 2017; 13, 3 (49): 263-268

DOI: https://doi.org/10.5114/aic.2017.70200

\section{Introduction}

Real-time 3D transesophageal echocardiography (TEE) with cardiac fluoroscopy $\mathrm{X}$-ray imaging is well introduced into practice and is used to monitor and assist catheter-based cardiac operations [1-6].

Cardiac fluoroscopy imaging is useful to monitor and control delivery tools into the desired site of the heart during transcatheter closure of paravalvular defects [3, $7,8]$. Fluoroscopy is superior to 3D TEE in visualization of the catheters, wires, devices, and calcified and metallic structures. The main disadvantages of fluoroscopy are the inability to determine the three-dimensional anatomy of intracardiac tissues, exposure to radiation and the need for contrast media.

3D TEE is a standard method to visualize intracardiac structural pathology and measure its hemodynamic significance. It is also used to control intracardiac manipulation of various instruments as well as paravalvular leak closure devices [8]. 3D TEE provides spatial intracardiac anatomy imaging in multiple planes, but its advantages are limited by echo shadowing, which reduces visualization of the catheters and metallic structures. Also experienced personnel are needed to achieve high quality 3D imaging and guide the procedures [7].

Merging these two imaging techniques combines the advantages of fluoroscopy and 3D TEE during paravalvular leak closure. As to date there are no studies comparing mitral paravalvular leak closure with or without fusion technology, we hypothesize that periprocedural fusion of cardiac fluoroscopy and real-time 3D TEE may make intracardiac procedures more controlled, precise and easier. Also it may reduce patients' and professionals' exposure to radiation [7].

We present three cases of transapical catheter-based mitral paravalvular leak closure. All patients were treated with devices specifically designed and manufactured by Occlutech Holding, Switzerland for paravalvlar leak closure. The Occlutech ${ }^{\circledR}$ Paravalvular Leak Device represents a novel device with unique rectangular- and squareshaped designs. Occlutech ${ }^{\circledR}$ Paravalvular Leak Device was CE marked in 2014 and is the first transcatheter device indicated and approved for aortic and mitral PVL closure. Procedures were performed with the assistance of fusion (EchoNavigator system, Phillips Medical Systems, Netherlands) of cardiac fluoroscopy imaging (Phillips AlluraClarity, Phillips Medical Systems, Netherlands) and real-time 3D TEE (Phillips Epiq7, Phillips Medical Systems, Netherlands).

\section{Procedure description}

The procedure was carried out in a hybrid operating room. Standard cardiac surgery monitoring was used. Prior to surgical draping, two self-adhesive external defibrillator pads were applied to the patient's back. With the patient in a supine position under general anesthesia and single lumen endotracheal intubation, left anterolateral thoracotomy $(5-6 \mathrm{~cm})$ was performed at the level of the left ventricle apex (preoperative TEE guidance). The pericardium was identified and opened. Blunt dissection of the adhesions was performed. Stay sutures were placed at both edges of the pericardium and hinged to the edges of the skin under the retractor. Two "U" shape sutures reinforced with Teflon pledgets

Corresponding author:

Aleksejus Zorinas, Heart Surgery Centre, Vilnius University Hospital Santariskiu Klinikos, Santariskiu 2, 08406 Vilnius, Lithuania, phone: +370 52365000, fax: +370 52365111, mobile: +370698 29079, e-mail: aleksejus.zorinas@santa.It

Received: 11.04.2017, accepted: 17.06.2017. 
$(5 \times 15 \mathrm{~mm})$ were placed and secured with the tourniquets perpendicular to each other at the apex of the heart. Then heparin was intravenously administered (150 U/kg), aiming for an activated clotting time of 250 to $300 \mathrm{~s}$. A needle puncture between four pledgets was performed and the guidewire was introduced into the left ventricle with subsequent short 6 Fr catheter sheath insertion.

Table I. Patients' demographic, clinical and procedural data

\begin{tabular}{|c|c|c|c|c|c|c|}
\hline Patients' data & Patient 1 & \multicolumn{2}{|c|}{ Patient 2} & \multicolumn{3}{|c|}{ Patient 3} \\
\hline Age [years] & 61 & \multicolumn{2}{|c|}{57} & \multicolumn{3}{|c|}{64} \\
\hline Gender & Female & \multicolumn{2}{|c|}{ Male } & \multicolumn{3}{|c|}{ Female } \\
\hline Mitral prosthesis description & $\begin{array}{c}27 \mathrm{~mm} \\
\text { Bileaflet } \\
\text { mechanical }\end{array}$ & \multicolumn{2}{|c|}{$\begin{array}{c}31 \mathrm{~mm} \\
\text { Bileaflet mechanical }\end{array}$} & \multicolumn{3}{|c|}{$\begin{array}{c}29 \mathrm{~mm} \\
\text { Bileaflet } \\
\text { mechanical }\end{array}$} \\
\hline 6 min walking distance $[\mathrm{m}]$ & 165 & \multicolumn{2}{|c|}{357} & \multicolumn{3}{|c|}{480} \\
\hline GFR $\left[\mathrm{ml} / \mathrm{min} / 1.73 \mathrm{~m}^{2}\right]$ & 46 & \multicolumn{2}{|c|}{79} & \multicolumn{3}{|c|}{78} \\
\hline Hemoglobin $[\mathrm{g} / \mathrm{l}]$ & 89 & \multicolumn{2}{|c|}{81} & \multicolumn{3}{|c|}{85} \\
\hline $\mathrm{LDH}[\mathrm{U} / \mathrm{l}]$ & 331 & \multicolumn{2}{|c|}{430} & \multicolumn{3}{|c|}{818} \\
\hline Haptoglobin [g/l] & 0.68 & \multicolumn{2}{|c|}{$<0.08$} & \multicolumn{3}{|c|}{$<0.08$} \\
\hline LVEF [\%] & 40 & \multicolumn{2}{|c|}{45} & \multicolumn{3}{|c|}{45} \\
\hline $\begin{array}{l}\text { Systolic pulmonary artery pressure } \\
{[\mathrm{mm} \mathrm{Hg}]}\end{array}$ & 65 & \multicolumn{2}{|c|}{50} & \multicolumn{3}{|c|}{42} \\
\hline EuroSCORE [\%] & 10.56 & \multicolumn{2}{|c|}{3.7} & \multicolumn{3}{|c|}{7.08} \\
\hline PVL dimensions [mm] & $9 \times 4$ & $8 \times 5$ & $2 \times 2$ & $4 \times 3$ & $2 \times 4$ & $3 \times 2$ \\
\hline $\begin{array}{l}\text { PVL location according to the clock and } \\
\text { segments of the mitral valve leaflets }\end{array}$ & $\begin{array}{l}3 \text { o'clock } \\
\text { (A3) }\end{array}$ & $\begin{array}{l}2 \text { o'clock } \\
\text { (A3) }\end{array}$ & $\begin{array}{l}6 \text { o'clock } \\
\text { (P2) }\end{array}$ & $\begin{array}{l}11 \text { o'clock } \\
\text { (A1) }\end{array}$ & $\begin{array}{l}2 \text { o'clock } \\
\text { (A3) }\end{array}$ & $\begin{array}{l}4 \text { o'clock } \\
\text { (P3) }\end{array}$ \\
\hline Severity of PVL regurgitation & Severe & Severe & Insignificant & Moderate & Mild & Mild \\
\hline Indications for PVL closure & $\begin{array}{l}\text { Heart failure } \\
\text { and anemia }\end{array}$ & & nia & & Anemia & \\
\hline \multicolumn{7}{|l|}{ Procedural data: } \\
\hline Occlutech PLD occluder article no. & 61PLD12W & 61PLD12W & Not closed & 60PLD5W & 60PLD4W & 62PLD3T \\
\hline Occluder waist size [mm] & $12 \times 5$ & $12 \times 5$ & & $5 \times 5$ & $5 \times 5$ & $3 \times 3$ \\
\hline $\begin{array}{l}\text { Size of the atrial [distal] disc } \\
\text { of the occluder [mm] }\end{array}$ & 21 & 21 & & $14 \times 14$ & $13 \times 13$ & $11.5 \times 11.5$ \\
\hline $\begin{array}{l}\text { Size of the ventricular (proximal) disc } \\
\text { of the occlude [mm] }\end{array}$ & 19 & 19 & & $12.5 \times 12$ & $11.5 \times 11.5$ & $10 \times 10$ \\
\hline Occluder type & Waist & Waist & & Waist & Waist & Twist \\
\hline Occluder shape & Rectangular & Rectangular & & Square & Square & Square \\
\hline Sheath size [F] & 9 & 9 & & 6 & 6 & 6 \\
\hline $\begin{array}{l}\text { Occluder delivery system Flex Pusher } \\
\text { item no. }\end{array}$ & $\begin{array}{l}\text { 50FP120 \& } \\
\text { 50FP120L }\end{array}$ & $\begin{array}{c}\text { 50FP120 \& } \\
\text { 50FP120L }\end{array}$ & & $\begin{array}{l}\text { 50FP100 \& } \\
\text { 50FP100L }\end{array}$ & $\begin{array}{l}\text { 50FP100 \& } \\
\text { 50FP100L }\end{array}$ & $\begin{array}{l}\text { 50FP100 \& } \\
50 F P 100 L\end{array}$ \\
\hline Occluder type & Waist & & & & & \\
\hline Cumulative fluoroscopy time & $8 \min 27 s$ & 36 & n $3 \mathrm{~s}$ & & $29 \min 46 \mathrm{~s}$ & \\
\hline Total cumulative air kerma [mGy] & 70.67 & & & & 157.7 & \\
\hline
\end{tabular}

GFR - glomerular filtration rate, LDH - lactate dehydrogenase, LVEF - left ventricular ejection fraction, PVL - paravalvular leak, PLD - a trade name for percutaneous trans-catheter device for paravalvular leak closure, atrade name for delivery system for paravalvular leak closure device. 
location, size, depth and shape of the paravalvular leak. The location of the paravalvular leak is marked in the EchoNavigator system to aid the pathway of the guidewire crossing the paravalvular leak [10]. A hydrophilic guidewire was used to pass through the defect with the help of a guiding catheter. The guiding catheter was advanced through the leak and replaced with a stiff wire. The delivery sheath was chosen according to the size of the occluder. The guiding catheter is removed and the delivery sheath is advanced through the defect. The paravalvular leak closure device was deployed under control of real-time 3D TEE and fluoroscopy. Position, orientation and hemodynamic effect of the closure device were checked. If the function of the prosthetic valve was not compromised, occluder discs were fully expanded and the paravalvular regurgitation closure device detached from the delivery system.

Following a successful paravalvular leak closure, catheters and sheaths were removed from the left ventricle and protamine sulfate administered. "U" shape sutures were securely tightened and the pericardium was closed with three or two interrupted sutures, leaving "communication" with the pleural space to allow blood to drain from the pericardial cavity. The pleural cavity was drained with one drain; usually it was inserted into one or two intercostal spaces below the incision. Patients were transferred to the cardiac intensive therapy unit and monitored for bleeding and hemodynamic instability. If no complication occurred patients were weaned from the ventilator, extubated and transferred to the ward on the next postoperative day.

\section{Patients treated}

Detailed descriptions of the patients' demographic, clinical and procedural data are presented in Table I.

\section{$1^{\text {st }}$ patient}

Thirteen years ago the 61-year-old woman underwent mitral valve replacement with a $27 \mathrm{~mm}$ bileaflet mechanical valve prosthesis and radiofrequency maze procedure for severe rheumatic mitral stenosis and long standing persistent atrial fibrillation. Aspiration of the pleural effusion was complicated by severe bleeding from the lung and was treated by revision through the right thoracotomy. The patient developed sepsis (Stenotrophomonas maltophilia) with subsequent severe mitral paravalvular leak. Four years later the patient underwent
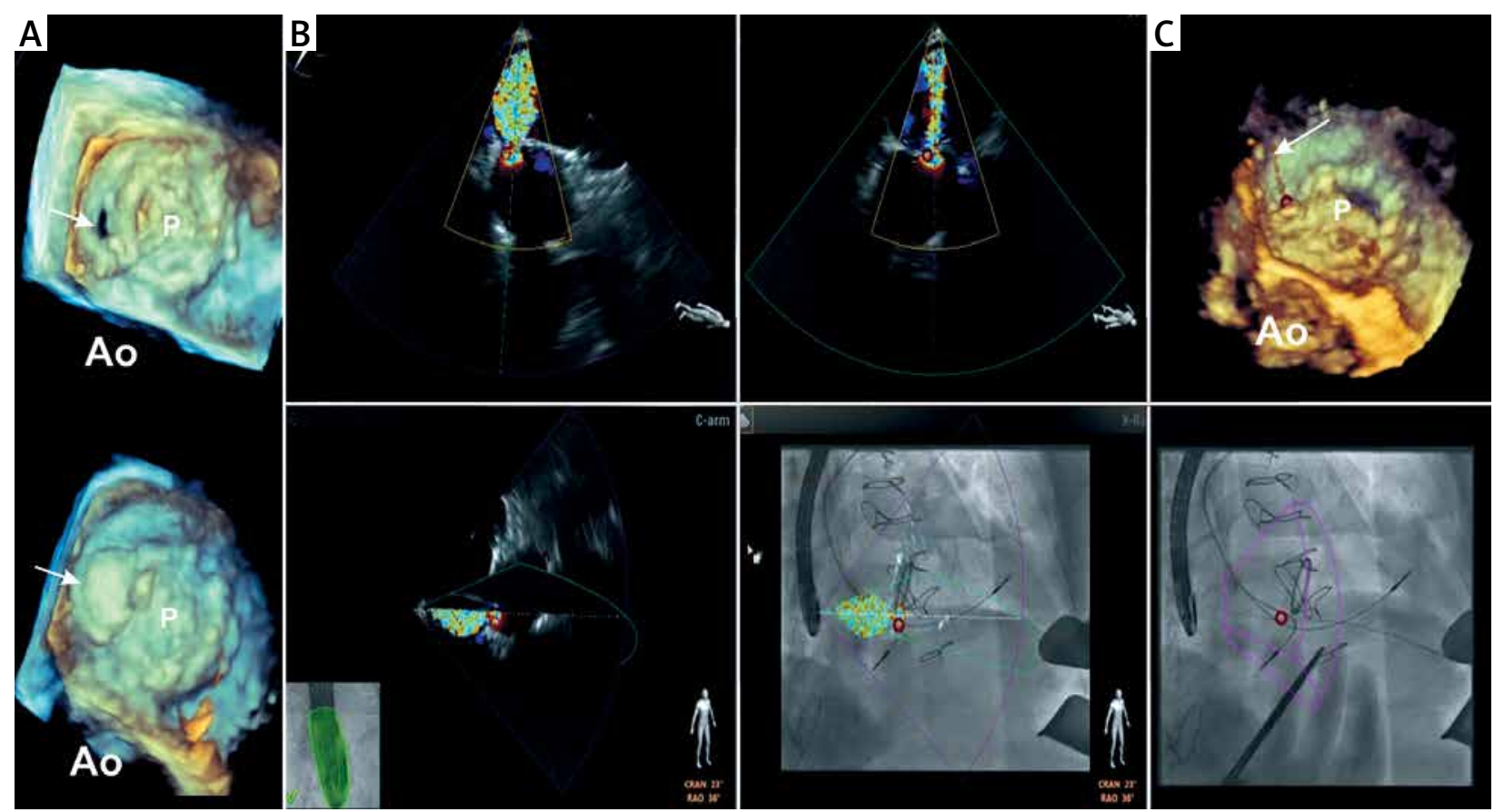

Figure 1. Patient 1 periprocedural fusion of fluoroscopy and real-time 3D TEE. A - Preprocedural (top) and postprocedural (bottom) 3D TEE images of the mitral prosthesis. The arrows point to the orifice of the paravalvular leak (PVL) located at 2 o'clock (surgical view) and the occluder position after the deployment. B - Four fused fluoroscopy and real-time TEE images. The X-plain color Doppler images of PVL regurgitant flow (top) are oriented according to $\mathrm{C}$-arm position (left bottom) and are fused with the fluoroscopy image (bottom). The location of PVL is marked by a red circle. C - Guidewire passage through the paravalvular defect. The fluoroscopy image (on the bottom) demonstrates wire crossing the red circle; 3D TEE image (top) confirms the guidewire passage through the defect. The arrow in the 3D TEE image shows the guidewire crossing the defect Ao-position of the aorta. 
redo surgery and mitral valve paravalvular leak suture repair through a right thoracotomy. Intraoperative TEE did not show any residual paravalvular leak. Unfortunately at discharge following that surgery on transthoracic echocardiography a paravalvular leak at the same location with severe mitral regurgitation was noted. On the basis of the patient's refusal, redo surgery was declined. For the following 9 years the patient was managed medically. With the patient's functional capacity gradually declining, she agreed to an elective transapical catheter-based procedure for mitral paravalvular leak.

The patient underwent an uneventful transapical catheter-based mitral paravalvular leak closure procedure (Figure 1). Immediate intraoperative hemodynamic improvement was noted; after closure of the paravalvular leak the central venous pressure decreased from $20 \mathrm{~mm} \mathrm{Hg}$ to $9 \mathrm{~mm} \mathrm{Hg}$, and systolic pulmonary artery (PA) pressure from $65 \mathrm{~mm} \mathrm{Hg}$ to $40 \mathrm{~mm} \mathrm{Hg}$.

The postoperative course was uneventful and the patient was discharged from hospital on the $11^{\text {th }}$ postoperative day. Discharge echo showed no paravalvular regurgitation and good left ventricle systolic function (LVEF > 55\%).

\section{$2^{\text {nd }}$ patient}

A 57-year-old man with previously known moderate aortic stenosis developed infective endocarditis of the aortic and mitral valves. He underwent aortic and mitral valve replacement with $21 \mathrm{~mm}$ and $31 \mathrm{~mm}$ bileaflet mechanical prostheses respectively. Three weeks after the operation the patient was diagnosed with severe anemia. The cause of anemia was established following transesophageal echocardiography which revealed severe mitral regurgitation due to paravalvular leak. The patient was scheduled for elective transapical catheter-based paravalvular defect closure.

He underwent an uneventful transapical catheter-based mitral paravalvular leak closure procedure (Figure 2) with complete closure of the defect. On intraoperative TEE no regurgitant flow across the occluded defect was noted. His postoperative course was uneventful. The patient was discharged on the $11^{\text {th }}$ postoperative day. Discharge echocardiography showed good left ventricle systolic function (LVEF > 50\%), systolic PA pressure of $55 \mathrm{~mm} \mathrm{Hg}$, trivial regurgitation through the occluded defect and trivial regurgitation through the untreated defect at 6 o'clock.
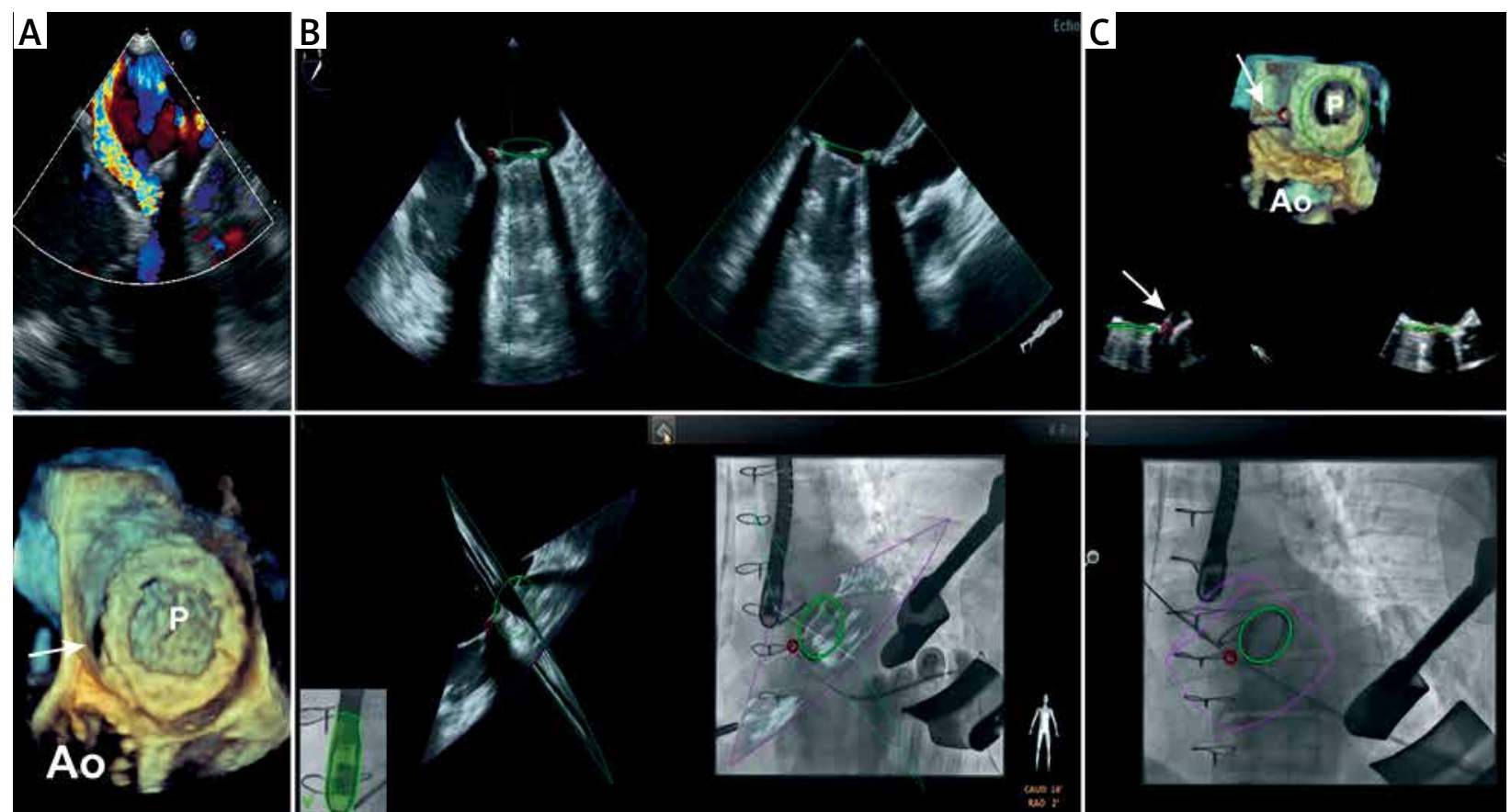

Figure 2. Patient 2 periprocedural fusion of fluoroscopy and real-time 3D TEE. A - The preprocedural color flow Doppler (top) and 3D images (bottom) demonstrates bileaflet mechanical mitral valve prostheses (P) and the paravalvular leak located at 2 o'clock (arrow). B - Two dimensional X-plain images (top), X-plain echo images are oriented according to $\mathrm{C}$-arm position (left bottom) and are fused with the fluoroscopy image (right bottom). Position of the prostheses is marked by a green circle in all images. The red circle identifies the location of the defect. C - Guidewire passage through the paravalvular defect. The fluoroscopy image (on the bottom) demonstrates wire crossing the red circle; 3D TEE image (top) confirms the guidewire passage through the defect. The arrow in the 3D TEE image shows the guidewire crossing the defect

Ao-position of the aorta. 


\section{$3^{\text {rd }}$ patient}

Two years ago a 64-year-old woman underwent aortic and mitral valve replacement with $25 \mathrm{~mm}$ and $29 \mathrm{~mm}$ mechanical prostheses respectively for rheumatic stenosis. Fourteen months after surgery she developed hemolytic anemia and presented with symptoms of general weakness. Transesophageal echocardiography revealed severe mitral regurgitation due to three paravalvular defects. For the 4 following months she was managed medically and 18 months after initial surgery the patient underwent a redo conventional operation and suture repair for mitral paravalvular leak. Unfortunately, discharge transthoracic echocardiography revealed reoccurrence of severe mitral paravalvular regurgitation. She was scheduled for elective transapical catheter-based mitral paravalvular leak closure a month later.

The patient underwent an uneventful transapical catheter-based mitral paravalvular leak closure procedure (Figure 3) with complete occlusion of all three defects. At discharge on transthoracic echocardiography no regurgitant flow across the occluded defects and good left ventricle systolic function were noted. The patient was discharged on the $6^{\text {th }}$ postoperative day with no postoperative complications.

\section{Comment}

In recent years, catheter-based closure of the mitral paravalvular leak has advanced globally. Nevertheless, due to the absence of standardized methods to quantify the regurgitation of the paravalvular leak, disagreement on the definition of success, tortuous anatomy of the defects and recent absence of dedicated devices for the closure of paravalvular leaks, the results of this method of treatment were debatable [8, 11-13]. The success rate of catheter-based paravalvular leak closure via the transapical approach reaches $100 \%$ compared to $66-78 \%$ in the trans-septal approach [11]. The closure of a mitral paravalvular leak using the transapical approach and real-time fluoroscopy and 3D TEE is efficient and safe [14]. Recent technological advances such as the ability to fuse fluoroscopy and real-time 3D TEE, as well as the development of devices dedicated to paravalvular leak closure, allow closure of a mitral paravalvular leak to be performed more conveniently $[8,15]$. Fusion is needed to guide the pathway of the pass through the paravalvular lesion. We have presented three cases of mitral paravalvular leak closure where this complex approach was applied. All three procedures showed technical and procedural success.
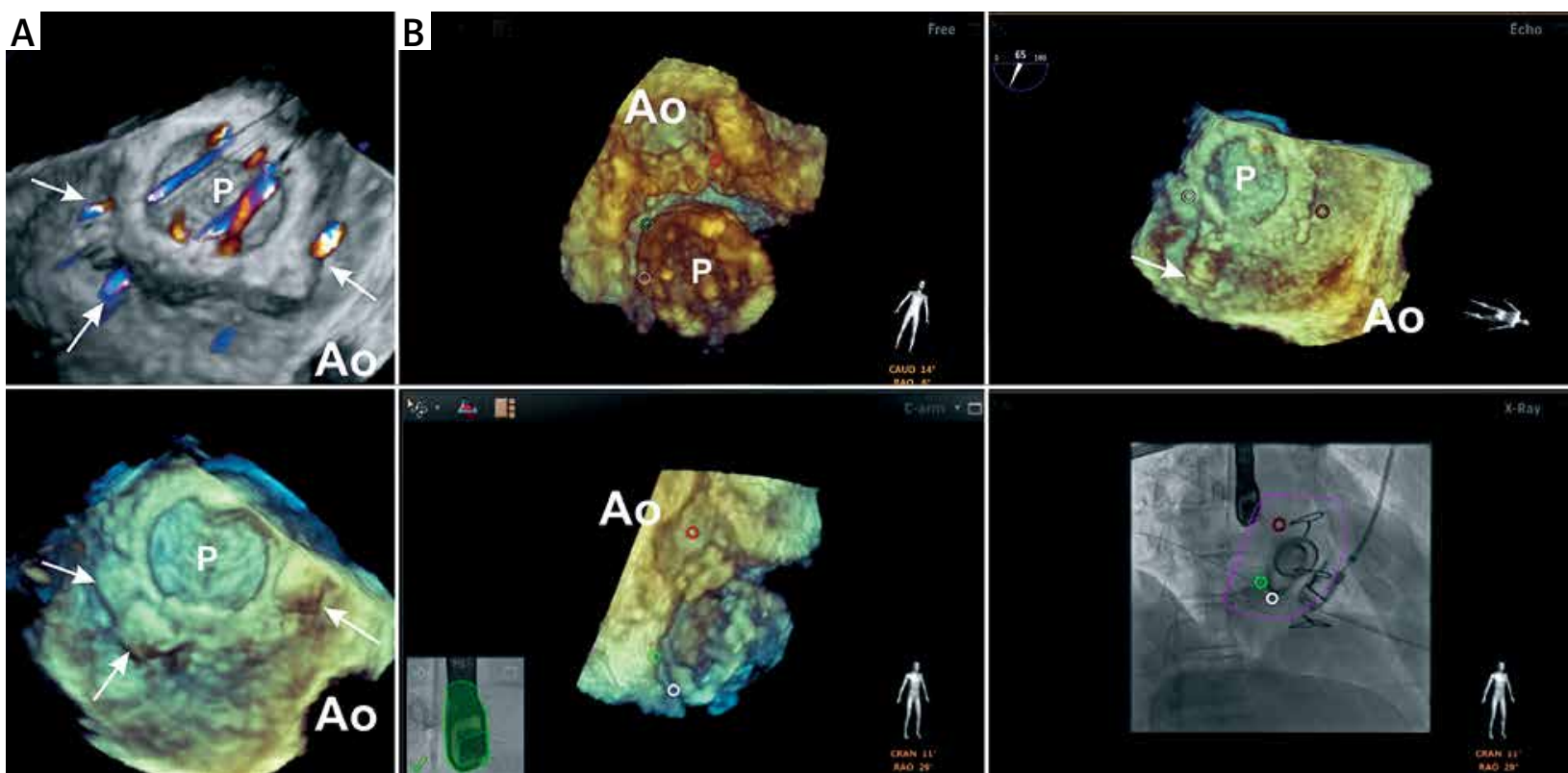

Figure 3. Patient 3 periprocedural fusion of fluoroscopy and real-time 3D TEE. A - The preprocedural 3D TEE color flow Doppler image (top) and 3D TEE image (bottom) with paravalvular defects closed with occluders. Arrows point to the defects. B - Real-time 3D TEE image (top left and bottom left) demonstrating ventricular perspective of the mitral prosthesis where colored (red, white, green) circles indicate paravalvular defects. Realtime 3D TEE image (top right) demonstrates atrial perspective of the mitral prosthesis where colored (red, white, green) circles indicate paravalvular defects. Fused fluoroscopy and real-time TEE image (bottom right) demonstrates wire crossing the green circle; 3D TEE image (top right) confirms the guidewire passage through the defect (arrow pointing to the guidewire)

Ao-position of the aorta. 


\section{Conclusions}

Fusion of fluoroscopy and real-time 3D TEE is a useful method in catheter-based paravalvular leak closure via the transapical approach.

\section{Conflict of interest}

Dr. Eustaquio Maria Onorato is a Consultant for Occlutech, manufacturer of the paravalvular leak closure device used and described in this publication. He also is Co-Principal Investigator for international, multi-center follow-up study monitoring the efficacy and safety of Occlutech PLD in patients with mitral or aortic paravalvular leak.

Others authors declare no conflict of interest.

\section{References}

1. Arribas-Jimenez A, Rama-Merchan JC, Barreiro-Perez M, et al. Utility of real-time 3-dimensional transesophageal echocardiography in the assessment of mitral paravalvular leak. Circ J 2016; 80: 738-44.

2. Biner S, Rafique AM, Kar S, Siegel RJ. Live three-dimensional transesophageal echocardiography-guided transcatheter closure of a mitral paraprosthetic leak by Amplatzer occluder. J Am Soc Echocardiogr 2008; 21: 1282 e7-9.

3. Epstein LM, Mitchell MA, Smith TW, Haines DE. Comparative study of fluoroscopy and intracardiac echocardiographic guidance for the creation of linear atrial lesions. Circulation 1998; 98: 1796-801.

4. Hamilton-Craig C, Boga T, Platts D, et al. The role of 3D transesophageal echocardiography during percutaneous closure of paravalvular mitral regurgitation. JACC Cardiovasc Imaging 2009; 2: 771-3.

5. Ozkan M, Gursoy OM, Astarcioglu MA, et al. Percutaneous closure of paravalvular mitral regurgitation with Vascular Plug III under the guidance of real-time three-dimensional transesophageal echocardiography. Turk Kardiyol Dern Ars 2012; 40: 632-41.

6. Roithinger FX, Steiner PR, Goseki Y, et al. Low-power radiofrequency application and intracardiac echocardiography for creation of continuous left atrial linear lesions. J Cardiovasc Electrophysiol 1999; 10: 680-91.

7. Arujuna AV, Housden RJ, Ma Y, et al. Novel system for real-time integration of 3-D echocardiography and fluoroscopy for image-guided cardiac interventions: preclinical validation and clinical feasibility evaluation. IEEE J Transl Eng Health Med 2014; 2: 1900110.

8. Balzer J, Zeus T, Hellhammer K, et al. Initial clinical experience using the EchoNavigator((R))-system during structural heart disease interventions. World J Cardiol 2015; 7: 562-70.

9. Gao G, Penney G, Ma Y, et al. Registration of 3D trans-esophageal echocardiography to X-ray fluoroscopy using image-based probe tracking. Med Image Anal 2012; 16: 38-49.

10. Biaggi P, Fernandez-Golfin C, Hahn R, Corti R. Hybrid imaging during transcatheter structural heart interventions. Curr Cardiovasc Imaging Rep 2015; 8: 33.

11. Millan X, Skaf S, Joseph L, et al. Transcatheter reduction of paravalvular leaks: a systematic review and meta-analysis. Can J Cardiol 2015; 31: 260-9.
12. Smolka G, Pysz P, Kozlowski M, et al. Transcatheter closure of paravalvular leaks using a paravalvular leak device - a prospective Polish registry. Adv Interv Cardiol 2016; 12: 128-34.

13. Ruiz CE, Hahn RT, Berrebi A, et al. Clinical trial principles and endpoint definitions for paravalvular leaks in surgical prosthesis: an expert statement. Eur Heart J 2017. doi:10.1093/eurheartj/ehx211.

14. Smolka G, Pysz P, Jasinski M, et al. Transapical closure of mitral paravalvular leaks with use of Amplatzer vascular plug III. J Invasive Cardiol 2013; 25: 497-501.

15. Goktekin O, Vatankulu MA, Ozhan H, et al. Early experience of percutaneous paravalvular leak closure using a novel Occlutech occluder. Eurolntervention 2016; 11: 1195-200. 